\title{
Use of a Fully Automated Internet-Based Cognitive Behavior Therapy Intervention in a Community Population of Adults With Depression Symptoms: Randomized Controlled Trial
}

Mark B Schure ${ }^{1,2}$, MS, PhD; Janet C Lindow ${ }^{2,3,4,5}, \mathrm{PhD} ;$ John H Greist ${ }^{2,3,6,7,8}$, MD; Paul A Nakonezny ${ }^{9,10}$, PhD; Sandra $\mathrm{J}_{\text {Bailey }}{ }^{1,2,11}$, PhD; William L Bryan ${ }^{2,12}$, PhD; Matthew J Byerly ${ }^{2,3,5,8}$, MD

${ }^{1}$ Department of Health \& Human Development, Montana State University, Bozeman, MT, United States

${ }^{2}$ Center for Mental Health Research and Recovery, Montana State University, Bozeman, MT, United States

${ }^{3}$ Department of Psychiatry, College of Medicine, University of Arizona, Tucson, AZ, United States

${ }^{4}$ Biomedical Research and Education Foundation of Southern Arizona, Tucson, AZ, United States

${ }^{5}$ Southern Arizona VA Health Care System, Tucson, AZ, United States

${ }^{6}$ School of Medicine and Public Health, University of Wisconsin, Madison, WI, United States

${ }^{7}$ Healthcare Technology Systems, Madison, WI, United States

${ }^{8}$ Department of Cell Biology and Neuroscience, Montana State University, Bozeman, MT, United States

${ }^{9}$ Department of Population and Data Science, University of Texas Southwestern Medical Center, Dallas, TX, United States

${ }^{10}$ Department of Psychiatry, University of Texas Southwestern Medical Center, Dallas, TX, United States

${ }^{11}$ Montana State University Extension, Bozeman, MT, United States

${ }^{12}$ One Montana, Bozeman, MT, United States

\section{Corresponding Author:}

Mark B Schure, MS, PhD

Department of Health \& Human Development

Montana State University

305 Herrick Hall

Bozeman, MT

United States

Phone: 14069943248

Email: mark.schure@montana.edu

\section{Abstract}

Background: Although internet-based cognitive behavior therapy (iCBT) interventions can reduce depression symptoms, large differences in their effectiveness exist.

Objective: The aim of this study was to evaluate the effectiveness of an iCBT intervention called Thrive, which was designed to enhance engagement when delivered as a fully automated, stand-alone intervention to a rural community population of adults with depression symptoms.

Methods: Using no diagnostic or treatment exclusions, 343 adults with depression symptoms were recruited from communities using an open-access website and randomized 1:1 to the Thrive intervention group or the control group. Using self-reports, participants were evaluated at baseline and 4 and 8 weeks for the primary outcome of depression symptom severity and secondary outcome measures of anxiety symptoms, work and social adjustment, psychological resilience, and suicidal ideation.

Results: Over the 8-week follow-up period, the intervention group ( $\mathrm{n}=181)$ had significantly lower depression symptom severity than the control group $(n=162 ; P<.001)$, with a moderate treatment effect size $(d=0.63)$. Moderate to near-moderate effect sizes favoring the intervention group were observed for anxiety symptoms $(P<.001 ; d=0.47)$, work/social functioning $(P<.001 ; d=0.39)$, and resilience $(P<.001 ; d=0.55)$. Although not significant, the intervention group was $45 \%$ less likely than the control group to experience increased suicidal ideation (odds ratio 0.55 ).

Conclusions: These findings suggest that the Thrive intervention was effective in reducing depression and anxiety symptom severity and improving functioning and resilience among a mostly rural community population of US adults. The effect sizes associated with Thrive were generally larger than those of other iCBT interventions delivered as a fully automated, stand-alone intervention. 
Trial Registration: ClinicalTrials.gov NCT03244878; https://clinicaltrials.gov/ct2/show/NCT03244878

(J Med Internet Res 2019;21(11):e14754) doi: $10.2196 / 14754$

\section{KEYWORDS}

internet-based cognitive behavior therapy; iCBT; depression symptoms; rural populations; RCT; randomized controlled trial; CBT

\section{Introduction}

Depression is the leading cause of disability globally [1] and is associated with impaired function [2], higher morbidity and mortality [3,4], greater health care use [5], and higher risk of suicide [6]. In the United States, the 12-month and lifetime prevalence of major depressive disorder is $10.4 \%$ and $20.6 \%$, respectively [2].

Cognitive behavior therapy (CBT), when delivered by clinicians, is an evidence-based psychotherapy for treating acute depression symptoms that also reduces relapse risk [7-9]. However, many barriers to receiving CBT exist, including geographic location, cost of insurance and care, long waitlists, and stigma for seeking treatment for mental illness [10]. For the approximately 46 million Americans living in rural regions [11], access to adequate mental health care is often limited or nonexistent [12]. In Montana (the focus of this study), $65.0 \%$ of residents live in rural regions [13]; the state also has a higher prevalence of depression than the national average and has had one of the highest suicide rates in the United States over the past four decades $[14,15]$. Despite the state's clear need for mental health services, $60 \%$ of the population live in mental health care professional shortage areas (defined as $<1$ psychiatrist/30,000) and only $13.3 \%$ of the total mental health provider need is met [12].

An attractive option for treating mental disorders in rural regions is the use of affordable, internet-delivered psychotherapy that requires little or no human intervention. Several studies of traditional face-to-face CBT and internet-based CBT (iCBT), herein defined as a class of online software apps that emulate in-person psychotherapy, have shown similar effectiveness for treating depression symptoms [16-18]. Additionally, a recent meta-analysis showed comparable effectiveness of iCBT interventions for reducing depression and anxiety symptoms among urban and rural populations outside the United States [19]. These results, combined with increasing broadband access in US rural communities [20], suggest that effective iCBT interventions have the potential for widespread public health impact by expanding the availability of low-cost, effective depression treatments [21,22] and providing an attractive alternative or complementary delivery strategy for populations that face the aforementioned barriers. However, no randomized controlled trials (RCTs) of iCBT interventions for depression have been conducted among US adult rural residents [21,22].

Although, in theory, the use of iCBT interventions to treat depression symptoms in US regions lacking sufficient mental health care services is an attractive treatment strategy, determining which iCBT intervention might be most effective is complicated by the considerable differences that exist in iCBT program design (eg, static text and pictures vs video-centric formats), support (fully automated to extensive human supports), adherence, and demonstrated effectiveness for treating depression symptoms in adults with a range of symptom severities (mild to severe) and diagnoses (none, major depressive disorder, unipolar affective disorder, or dysthymia) [10,21,22]. Additionally, participant engagement has been a significant challenge for some depression iCBT interventions. Fully automated, stand-alone iCBT interventions generally have higher treatment dropout rates $(74 \%)$ than those provided with therapist or administrative support (28\% and $38 \%$, respectively) [23]. Lower engagement likely decreases the effectiveness of iCBT interventions for depression. For example, in three studies that reported participants completing fewer than three mean intervention sessions, two found no significant difference in depression symptoms for the intervention compared to the control [24,25] and the third found a small effect size (0.26) [26] for depression symptoms. To date, six of the seven studies of fully automated, stand-alone versions of iCBT have demonstrated small-to-medium clinical effects [26-31] and one found no significant effects [25]. Thus, improvements in iCBT interventions and delivery strategies are needed.

The intervention evaluated in this study, Thrive, is a fully automated, stand-alone iCBT intervention designed to reduce depression symptoms using a video-based platform. The intervention incorporates classic cognitive behavior therapy themes in modules on Constructive Thinking (Cognitive Restructuring) [32], Pleasant Activities (Behavioral Activation) $[33,34]$, and Assertive Communication (Social Skills Training) [35]. Each module consists of 10 lessons, and there is an introduction lesson that offers suggestions for choice of a first module (31 total lessons). Within each module, Thrive's algorithms personalize content, exercises, and recommendations for participants based on their input and progress. A pilot feasibility study of Thrive in a US primary health care setting included 37 patients with depression (Symptom Checklist-20 score $>1.75)$, of whom $59 \%$ (22/37) had suicidal thoughts at baseline (Symptom Checklist-20 item 13) [36]. At the 4-month follow-up, $52 \%$ (16/31) had $\geq 50 \%$ reduction in depression, $46 \%$ $(14 / 31)$ had a clinically significant decrease in depression symptoms, and fewer reported having recent suicidal thoughts (35\% vs $59 \%$ at baseline) [36]. Thrive was also offered to employees of four businesses located in four states. A total of 227 individuals with baseline depression symptom severity (Patient Health Questionnaire-9 item [PHQ-9] [37]) scores between 5 and 27 (mean score 10.5) and at least one follow-up PHQ-9 score logged into Thrive an average of 10.5 times and experienced an average PHQ-9 improvement of 4.4 points (42\%) over an average of 7 weeks (B. Coleman, personal communication, 3 Sept 2019). Thus, preliminary data suggested that Thrive might be efficacious. Given the lack of evidence of 
iCBT depression interventions in rural US settings, the primary objective of this RCT was to evaluate the short-term effectiveness of Thrive to reduce depression symptom severity when delivered directly to a community population of adults with depression symptoms living in Montana, one of the least densely populated states in the United States [38]. Because the Thrive intervention required no clinician interaction or support for delivery and assessments, findings are likely predictive of the use and impact of the Thrive intervention in real-world settings and support the promise of iCBT to address unmet mental health care needs in rural US regions and possibly beyond.

\section{Methods}

\section{Trial Design}

An RCT compared the efficacy of the fully automated, stand-alone Thrive intervention to a waitlist control (WLC) in reducing depression symptoms among adults. Participants were recruited from communities across Montana and immediately randomized 1:1 to the intervention group (Thrive) or a WLC group (delayed access to Thrive until the 8-week follow-up assessment) after meeting the inclusion criteria and providing electronic informed consent on the study website [39] (Multimedia Appendix 1). Participants in both groups were assessed for primary and secondary outcomes at baseline and 4 and 8 weeks postenrollment. Enrollment occurred between September 2017 and January 2018, and all assessments were completed by March 2018. The Montana State University Institutional Review Board approved the protocol and all related materials (\#MS033017-FC) prior to study initiation. The study is registered at ClinicalTrials.gov (NCT03244878).

\section{Participants}

Participants were recruited using community fliers, public service announcements, local newspaper advertisements, newsletters, and community social media sites. The study was also promoted through select state organization email listservs, large employers, local health care providers, Facebook, a Craigslist community page, community meeting events, and Montana State University Extension faculty communications with their respective counties. All methods directed potential participants to a study website, which informed potential applicants about the study expectations; determined their eligibility; and guided those eligible through the informed consent, randomization, and assessment process [39].

Inclusion criteria included adults aged $\geq 18$ years with mild-to-severe depression severity (PHQ-9 score >5) [37]; Montana residency; a valid email address; and regular access to broadband internet via a computer, tablet, or smartphone. At enrollment, potential participants who indicated recent suicidal ideation (PHQ-9 item 9 score $>0$ ) were asked to confirm that they could stay safe and those responding that they could not were considered ineligible (see the safety protocol description below for the handling of cases of suicidal ideation and Multimedia Appendix 4). All participants provided electronic informed consent prior to study participation. All participants were informed that they were free to obtain and use any additional care available throughout their participation in the trial. In total, 463 participants were enrolled, and of these, 109 were deemed fraudulent identities and their data were removed prior to analyses (Multimedia Appendix 2). Additionally, three individuals provided invalid email addresses, which led to their exclusion from study participation, and data from two control participants were discarded, as they were accidentally provided access to the intervention immediately. Six participants were excluded because of missing baseline data on the covariate "currently receiving psychosocial therapy for depression," which was required for data analysis. The final analytic sample included 343 participants (intervention: $n=181$; control: $n=162$ ), of which $86(25 \%)$ participants did not complete any follow-up assessments (Multimedia Appendices 2 and 3).

\section{Intervention Group}

Thrive is a fully automated, stand-alone, individually tailored iCBT intervention for depression developed by Waypoint Health Innovations [40]. Participants accessed the intervention with a Web browser or mobile app. Intervention content is largely delivered by video with minimal text and employs three structured interactive modules focused on behavioral activation, cognitive restructuring, and social skills training CBT techniques. Based on user input and usage patterns, the intervention uses algorithms to personalize feedback and tailor user progression through each therapeutic modality. Based on the study results from a qualitative study on the acceptability of Thrive [41], 6 of the 320 videos were replaced with videos depicting scenarios and settings characteristic of rural Montana to enhance engagement among the study population.

\section{Control Group}

The control group received a link to general depression information at the National of Institute of Mental Health [42]. Participants in the control group were also provided a link to Montana's National Alliance on Mental Illness resource page [43]. The control group was granted access to Thrive after completing the 8-week assessment.

\section{Assessments}

Participants completed study assessments on the study website at baseline, 4 weeks, and 8 weeks. Automated email reminders were sent when each assessment was due, with two additional follow-up automated reminder emails sent within 7 days to those who had not completed the assessment. If the participant did not complete the scheduled assessment within a 10-day window, data were considered lost to follow-up for that assessment (Multimedia Appendix 10). A US \$25 Amazon gift code was sent following completion of each assessment.

\section{Outcomes}

The primary outcome measure was participants' self-reported depression symptom severity PHQ-9 score. Prespecified secondary outcomes included anxiety symptom severity, daily functioning, and resilience. Suicidal ideation was added as a secondary outcome after the original protocol was developed, but prior to the start of analyses.

\section{Measures}

All outcomes were assessed using self-reported, validated measures automatically administered via the study website. 
Depression symptom severity was evaluated using the PHQ-9, which incorporates the major depressive disorder "A criterion" symptoms of Diagnostic and Statistical Manual of Mental Disorders (DSM)-IV and DSM-5 (score range: 0-27, with higher scores indicating worse symptoms) [37]. Anxiety symptom severity was measured with the Generalized Anxiety Disorder Scale (GAD-7, score range: 0-21), with higher scores indicating worse symptoms [44]. Daily functioning was measured using the Work and Social Adjustment Scale (WSAS, score range: 0-40), with higher scores indicating a greater impact of depression on daily functioning [45]. The abbreviated version of the Conner-Davidson Resilience Scale (CD-RISC-10) measured resilience (score range: 0-40), with higher scores indicating greater resilience [46]. Lastly, suicidal ideation was assessed using item 9 of the PHQ-9 measure ("Thoughts that you would be better off dead or of hurting yourself" in the past two weeks). Item 9 was treated as an ordinal scale that ranged from 0 ("not at all," no suicidal ideation) to 3 ("nearly every day") [37].

\section{Safety}

When study applicants endorsed having at least some suicidal ideation (PHQ-9 item 9 score $>0$ ) at study enrollment, the study website displayed multiple sources of immediate help (Multimedia Appendix 4) and asked applicants to declare whether they were sure they could stay safe. Applicants answering that question negatively were considered ineligible to participate in the study. Notably, no applicants reported that they could not stay safe. Regardless of the response to the "stay safe" question, the study website prompted the individual to seek help and provided the same sources of help described in Multimedia Appendix 4. If an enrolled participant indicated s/he had at least some suicidal ideation during the 4- or 8-week assessments (PHQ-9 item 9 score >0), the study website immediately prompted the individual to seek help and provided the same sources of help. Additionally, the Thrive intervention directed individuals with PHQ-9 scores $\geq 20$ at any assessment and those with PHQ-9 scores $\geq 10$ on the third assessment to seek help from a doctor. A thorough description of all safety measured used is described in Multimedia Appendix 4. Participants were provided email addresses (lead investigator and IRB Chair) for reporting of any adverse experiences or events.

\section{Covariates}

An initial pool of 10 variables was selected $a$ priori for analysis as potential covariates of depression severity, anxiety, functional impairment, resilience, and suicidal ideation (Multimedia Appendix 5).

\section{Statistical Analysis}

Outcomes were assessed at baseline and at 4 and 8 weeks. The change over time in each continuous outcome and suicidal ideation was compared between the intervention and control groups using a linear mixed model analysis of repeated measures and an ordinal logistic regression model within a Generalized Estimating Equation framework, respectively. A separate model was conducted on each outcome measure. Each model contained fixed-effects terms for treatment (intervention vs control), time, treatment $\times$ time interaction, and respective baseline measure (prior to the intervention) as covariates. Receiving therapy for depression (yes/no) at the baseline assessment was also included as a covariate in each model. Least squares means (adjusted treatment means) and adjusted odds ratios (OR) were estimated as part of the mixed model and ordinal logistic model, respectively, to interpret the treatment effect. For the ordinal logistic regression, the cumulative probabilities were modeled over the higher-ordered suicidal ideation scale scores (more suicidal ideation).

Statistical analyses were performed using SAS software, version 9.4 (SAS Institute, Inc, Cary, North Carolina). Maximum likelihood estimators allow efficient parameter estimation using only available data under an assumption of missing at random [47-49]. The level of significance was set at $\alpha=0.05$ (two-tailed), and the Bonferroni method was implemented to control false-positives over the multiple tests. The $95 \%$ CIs for the point estimates of treatment group effects were also adjusted to match the Bonferroni adjustment to significance levels in the corresponding test. A priori evaluable sample size for a statistical power of $80 \%$ was estimated (Multimedia Appendix $6)$.

\section{Results}

\section{Participant Characteristics}

Participant baseline characteristics are described in Table 1. The cohort was predominately female $(290 / 343,85 \%)$ and Caucasian (319/343, 93\%), with a mean age of 42.9 (SD 13.3) years. Most participants were married (198/343, 58\%); employed, or in school $(265 / 343,77 \%)$; had some higher education (318/343, 93\%); and lived in rural regions (287/343, $84 \%)$. In addition, 59\% (202/343) of participants were receiving medication, psychosocial therapy, or other treatment for depression or anxiety at baseline. No significant differences for any outcome measure were observed at baseline (Table 2) nor did the baseline depression symptom severity differ between groups (Multimedia Appendix 7). Participants randomized to the intervention group completed a mean of 8.7 lessons. No adverse experiences or adverse events were reported by either study group. 
Table 1. Baseline characteristics in the intent-to-treat population.

\begin{tabular}{|c|c|c|c|}
\hline Characteristics & Total sample $(\mathrm{N}=343), \mathrm{n}(\%)$ & iCBT $^{\mathrm{a}} \operatorname{group}^{\mathrm{b}}(\mathrm{n}=181), \mathrm{n}(\%)$ & Control group $(\mathrm{n}=162), \mathrm{n}(\%)$ \\
\hline Age, mean (SD) & 42.9 (SD 13.3) & $42.1(12.8)$ & $43.8(13.8)$ \\
\hline Female & $290(85.0)$ & $160(88.4)$ & $130(81.2)$ \\
\hline \multicolumn{4}{|l|}{ Race } \\
\hline White & $319(93.0)$ & $170(93.9)$ & $149(92.0)$ \\
\hline Other & $24(7.0)$ & $11(6.1)$ & $13(8.0)$ \\
\hline \multicolumn{4}{|l|}{ Marital status } \\
\hline Single & $145(42.3)$ & $78(43.1)$ & $67(41.4)$ \\
\hline Married/domestic partnership & $198(57.7)$ & $103(56.9)$ & $95(58.6)$ \\
\hline \multicolumn{4}{|l|}{ Employment status } \\
\hline Employed or student & $265(77.3)$ & $136(75.1)$ & $129(79.6)$ \\
\hline Retired or unemployed & $78(22.7)$ & $45(24.9)$ & $33(20.4)$ \\
\hline Veteran & $15(4.4)$ & $7(3.9)$ & $8(4.9)$ \\
\hline \multicolumn{4}{|l|}{ Education } \\
\hline High school degree or less & $25(7.3)$ & $10(5.5)$ & $15(9.3)$ \\
\hline $\begin{array}{l}\text { Some college, bachelor or associate degree, } \\
\text { or trade school }\end{array}$ & $236(68.8)$ & $129(71.3)$ & $107(66.0)$ \\
\hline Graduate or professional degree & $82(23.9)$ & $42(23.2)$ & $40(24.7)$ \\
\hline \multicolumn{4}{|l|}{ Health insurance } \\
\hline Private & $244(71.1)$ & $140(77.3)$ & $104(64.2)$ \\
\hline Public & 74 (21.6) & $32(17.7)$ & $42(25.9)$ \\
\hline Other & $9(2.6)$ & $5(2.8)$ & $4(2.5)$ \\
\hline None & $16(4.7)$ & $4(2.2)$ & $12(7.4)$ \\
\hline \multicolumn{4}{|l|}{ Rural classification $^{c}$} \\
\hline Urban & $56(16.3)$ & $27(14.9)$ & $29(17.9)$ \\
\hline Large rural & $73(21.3)$ & $42(23.2)$ & $31(19.1)$ \\
\hline Small rural & $116(33.8)$ & $60(33.2)$ & $56(34.6)$ \\
\hline Isolated & 98 (28.6) & $52(28.7)$ & $46(28.4)$ \\
\hline \multicolumn{4}{|l|}{ Receiving mental health treatment ${ }^{d}$} \\
\hline Yes & $202(58.9)$ & $103(56.9)$ & 99 (61.1) \\
\hline No & $141(41.1)$ & $78(43.1)$ & $63(38.9)$ \\
\hline
\end{tabular}

aCBT: internet-based cognitive behavior therapy.

$\mathrm{b}_{\text {Thrive intervention group. }}$

${ }^{\mathrm{c}}$ Defined using rural urban commuting area codes [50].

${ }^{\mathrm{d}}$ Defined as receiving any clinical care or taking medication(s) for depression symptoms. 
Table 2. Baseline clinical measures for total sample and treatment groups.

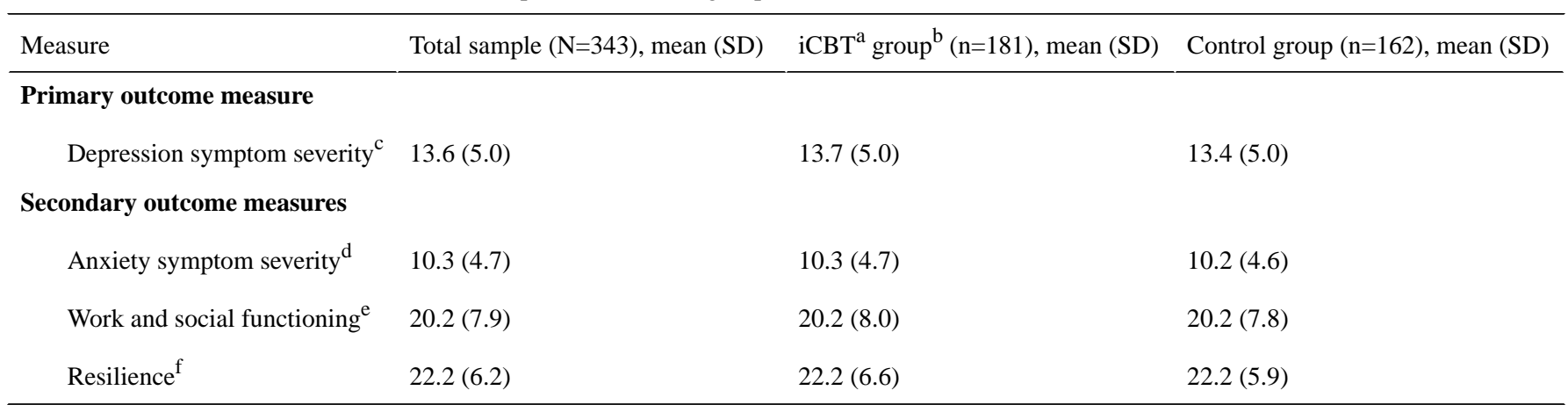

aCBT: internet-based cognitive behavior therapy.

$\mathrm{b}_{\text {Thrive intervention group. }}$

${ }^{\mathrm{c}}$ Patient Health Questionnaire-9 score range=0-27.

${ }^{\mathrm{d}}$ Generalized Anxiety Disorder Scale 7-Item score range=0-21.

${ }^{\mathrm{e}}$ Work and Social Adjustment Scale score range=0-40.

${ }^{\mathrm{f}}$ Connor-Davidson Resilience Scale 10-Item score range $=0-40$.

\section{Clinical Outcomes}

\section{Primary Outcome}

Significant main effects of treatment $\left(F_{1,248}=28.67\right.$; raw $P<.001$, adjusted $P<.001)$ and time $\left(F_{1,216}=11.94\right.$; raw $P<.001$, adjusted $P<.001$ ), favoring the Thrive intervention, were observed for depression symptom severity (Table 3 ), and a moderate treatment effect size (Cohen $d=0.63, P<.001$; Table 4) was

found. The pattern of the overall least squares treatment group means showed that depression severity (following 8 weeks of intervention) was significantly lower for the intervention group than for the control group (7.702 [SE 0.336] vs 10.224 [SE 0.328 ], raw $P<.001$, adjusted $P<.001 ; d=0.63$; Table 4$)$. The same pattern was observed with simple treatment group effects at weeks 4 and 8 (raw $P<.001$, adjusted $P<.001$; PHQ-9, Table 4).

Table 3. Main effects of treatment, time, and treatment by time interaction effects from the mixed model and ordinal logistic regression analysis for depression symptom severity, anxiety symptom severity, work/social functioning, resilience, and suicidal ideation.

\begin{tabular}{|c|c|c|c|c|c|c|c|c|c|c|}
\hline \multirow[t]{2}{*}{ Covariates and effects } & \multicolumn{2}{|c|}{$\begin{array}{l}\text { Depression symptoms } \\
\left(\text { PHQ-9 }{ }^{\text {a }}\right)\end{array}$} & \multicolumn{2}{|c|}{$\begin{array}{l}\text { Anxiety symptoms } \\
\left(\mathrm{GAD}-7^{\mathrm{b}}\right)\end{array}$} & \multicolumn{2}{|c|}{ Functioning $\left(W_{S A S}{ }^{c}\right)$} & \multicolumn{2}{|c|}{$\begin{array}{l}\text { Resilience (CD-RISC- } \\
10^{\mathrm{d}} \text { ) }\end{array}$} & \multicolumn{2}{|c|}{$\begin{array}{l}\text { Suicidal ideation } \\
\text { (PHQ-9) }\end{array}$} \\
\hline & $\begin{array}{l}F \text { statistic } \\
(d f)\end{array}$ & $P$ value & $\begin{array}{l}F \text { statistic } \\
(d f)\end{array}$ & $P$ value & $\begin{array}{l}F \text { statistic } \\
(d f)\end{array}$ & $P$ value & $\begin{array}{l}F \text { statistic } \\
(d f)\end{array}$ & $P$ value & $\chi^{2}(d f)$ & $P$ value \\
\hline Baseline outcome $\mathrm{e}^{\mathrm{e}}$ & $\begin{array}{l}F(1,245) \\
=119.76\end{array}$ & $<.001$ & $\begin{array}{l}F(1,253.6) \\
=133.56\end{array}$ & $<.001$ & $\begin{array}{l}\mathrm{F}(1,251.7) \\
=181.17\end{array}$ & $<.001$ & $\begin{array}{l}\mathrm{F}(1,247.7) \\
=323.87\end{array}$ & $<.001$ & $67.18(1)$ & $<.001$ \\
\hline Therapy ${ }^{\mathrm{f}}$ & $\begin{array}{l}F(1,249) \\
=7.58\end{array}$ & .006 & $\begin{array}{l}\mathrm{F}(1,254.1) \\
=8.29\end{array}$ & .004 & $\begin{array}{l}F(1,250.7) \\
=8.82\end{array}$ & .003 & $\begin{array}{l}F(1,243.7) \\
=4.17\end{array}$ & .04 & $3.71(1)$ & .054 \\
\hline \multicolumn{11}{|l|}{ Effects } \\
\hline Treatment $^{\mathrm{g}}$ & $\begin{array}{l}\mathrm{F}(1,248) \\
=28.67\end{array}$ & $\begin{array}{l}<.001 \\
<.001^{\mathrm{h}}\end{array}$ & $\begin{array}{l}F(1,252.8) \\
=16.14\end{array}$ & $\begin{array}{l}<.001 \\
<.001^{\mathrm{h}}\end{array}$ & $\begin{array}{l}\mathrm{F}(1,249.4) \\
=11.09\end{array}$ & $\begin{array}{l}.001 \\
.005^{\mathrm{h}}\end{array}$ & $\begin{array}{l}F(1,243.6) \\
=22.71\end{array}$ & $\begin{array}{l}<.001 \\
<.001^{\mathrm{h}}\end{array}$ & $2.74(1)$ & $\begin{array}{l}.098 \\
.49^{\mathrm{h}}\end{array}$ \\
\hline Time (weeks) & $\begin{array}{l}\mathrm{F}(1,216) \\
=11.94\end{array}$ & $\begin{array}{l}<.001 ; \\
<.001^{\mathrm{h}}\end{array}$ & $\begin{array}{l}F(1,221) \\
=4.68\end{array}$ & $\begin{array}{l}.03 ; \\
.16^{\mathrm{h}}\end{array}$ & $\begin{array}{l}\mathrm{F}(1,217.5) \\
=0.49\end{array}$ & $\begin{array}{l}.49 \\
>.99^{h}\end{array}$ & $\begin{array}{l}\mathrm{F}(1,213.3) \\
=5.82\end{array}$ & $\begin{array}{l}.02 \\
.08^{\mathrm{h}}\end{array}$ & $5.19(1)$ & $\begin{array}{l}.02 \\
.11^{\mathrm{h}}\end{array}$ \\
\hline Treatment $\times$ time & $\begin{array}{l}F(1,216) \\
=0.12\end{array}$ & $\begin{array}{l}.73 \\
>.99^{h}\end{array}$ & $\begin{array}{l}F(1,221) \\
=0.43\end{array}$ & $\begin{array}{l}.51 \\
>.99^{\mathrm{h}}\end{array}$ & $\begin{array}{l}F(1,217.5) \\
=3.66\end{array}$ & $\begin{array}{l}.06 \\
.29^{\mathrm{h}}\end{array}$ & $\begin{array}{l}F(1,213.3) \\
=0.22\end{array}$ & $\begin{array}{l}.64 \\
>.99^{h}\end{array}$ & $1.39(1)$ & $\begin{array}{l}.24 \\
>.99^{h}\end{array}$ \\
\hline
\end{tabular}

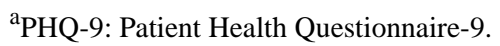

${ }^{\mathrm{b}}$ GAD-7: Generalized Anxiety Disorder 7-Item scale.

${ }^{\mathrm{c}}$ WSAS: Work and Social Adjustment Scale.

${ }^{\mathrm{d}}$ CD-RISC-10: Connor-Davidson Resilience Scale 10-Item

${ }^{\mathrm{e}}$ Baseline scores for PHQ-9, GAD-7, WSAS, and CD-RISC-10.

${ }^{\mathrm{f}}$ Receiving treatment for depression at baseline.

$\mathrm{g}_{\text {Thrive iCBT intervention or control. }}$

${ }^{\mathrm{h}} P$ values adjusted by the Bonferroni method. 
Table 4. Effect of the intervention on depression severity, anxiety severity, work/social functioning, and resilience.

\begin{tabular}{llll}
\hline Outcome and group & $\begin{array}{l}\text { Week 4, LSM }{ }^{\mathrm{a}}(\mathrm{SE}), \\
(95 \% \mathrm{CI})\end{array}$ & $\begin{array}{l}\text { Week 8, LSM (SE), } \\
(95 \% \mathrm{CI})\end{array}$ & $\begin{array}{l}\text { Overall timed-average } \\
(\text { weeks } 4-8), \mathrm{LSM}(\mathrm{SE}), \quad(\text { weeks } 4-8) \\
(95 \% \mathrm{CI})\end{array}$
\end{tabular}

$F$ statistic $(d f) \quad P$ value $\quad$ Cohen $d$

\section{Depression severity (PHQ-9 ${ }^{\text {b }}$ )}

Intervention group

Control group

LSM group difference N/A

\section{Anxiety severity (GAD-7 $\mathbf{7}^{\mathbf{f}}$ )}

Intervention group

Control group

LSM group difference

$\begin{array}{ll}8.165(0.373), & 7.240(0.385), \\ \text { (7.430 to } 8.899) & (6.481 \text { to } 7.998) \\ 10.602(0.371), & 9.845(0.367), \\ (9.873 \text { to } 11.33) & (9.122 \text { to } 10.568) \\ \text { N/A } & \text { N/A }\end{array}$

N/A

$6.481(0.358)$

(5.777 to 7.185 )

$8.382(0.341)$

(7.710 to 9.053 )

N/A

15.407 (0.621),
$(14.185$ to 16.629$)$
$18.682(0.592)$,
$(17.517$ to 19.848$)$
N/A

LSM group difference

N/A
$7.702(0.336)$

(7.039 to 8.365 )

$10.224(0.328)$

(9.576 to 10.871 )

$-2.521(0.471)$,

(-3.448 to -1.593$)$

$(-3.743 \text { to }-1.298)^{\mathrm{d}}$

$6.801(0.312)$

(6.186 to 7.415 )

8.553 (0.304),

(7.953 to 9.153 )

-1.752 (0.436),

( -2.610 to -0.893$)$,

$(-2.883 \text { to }-0.620)^{\mathrm{d}}$

$15.920(0.542)$,

(14.852 to 16.988 )

$18.443(0.529)$,

(17.401 to 19.486$)$

-2.523 (0.757),

(-4.016 to -1.031$)$,

$(-4.487 \text { to }-0.558)^{\mathrm{d}}$

$25.273(0.341)$ (24.601 to 25.945$)$

23.002 (0.332),

(22.346 to 23.657)

2.271 (0.476),

(1.332 to 3.209),

$(1.035 \text { to }-3.506)^{\mathrm{d}}$

$\begin{array}{lll}\text { N/A } & \text { N/A } & \text { N/A } \\ \text { N/A } & \text { N/A } & \text { N/A } \\ & & \\ \text { F }(1,248)=28.67 & <.001 ; & 0.628 \\ & <.001^{\mathrm{e}} & \end{array}$

N/A N/A N/A

N/A N/A N/A

$\mathrm{F}(1,252.8)=16.14 \quad<.001 ; \quad 0.470$

$<.001^{\mathrm{e}}$

N/A

N/A N/A

N/A N/A N/A

$F(1,249.4)=11.09 \quad .001 ; \quad 0.389$

$<.005^{\mathrm{e}}$

N/A N/A N/A

N/A N/A N/A

$\mathrm{F}(1,243.6)=22.71 \quad<.001 ; \quad 0.552$ $<.001^{\mathrm{e}}$

${ }^{\mathrm{a}}$ LSM: least squares means.

${ }^{b}$ PHQ-9: Patient Health Questionnaire-9.

${ }^{\mathrm{c}} \mathrm{N} / \mathrm{A}$ : not applicable.

${ }^{\mathrm{d}}$ Bonferroni-adjusted $95 \%$ CIs.

${ }^{\mathrm{e}} P$ values adjusted by the Bonferroni method. The adjusted $P$ value was associated with the test (F statistic) of the overall timed-average difference of the LSM estimate between the groups (Thrive intervention vs control).

${ }^{\mathrm{f}}$ GAD-7: Generalized Anxiety Disorder 7-Item scale.

${ }^{\mathrm{g}}$ WSAS: Work and Social Adjustment Scale.

${ }^{\mathrm{h}}$ CD-RISC-10: Connor-Davidson Resilience Scale 10-Item. 


\section{Secondary Outcomes}

The results of the main effects (treatment and time) as well as the treatment by time interaction effect from the mixed model and ordinal logistic regression analysis for the secondary outcomes of anxiety, functional impairment, resilience and suicidal ideation are reported in Table 3. Anxiety symptom severity, work and social functional impairment, and resilience were significantly improved for the Thrive intervention group compared to the control group (raw $P<.001$, adjusted $P<.001$ for all; $d=0.47, d=0.39$, and $d=0.55$, respectively; Table 4 ). This pattern was also observed with simple treatment group effects at weeks 4 and 8 (Multimedia Appendix 8).

Suicidal ideation was reported by $41 \%, 19 \%$, and $16 \%$ of participants at baseline, week 4 , and week 8 , respectively. The predicted odds of increased suicidal ideation (PHQ-9 item 9) for the intervention group showed a lower trend than that of the control group, but did not reach significance (OR 0.55, 95\% CI 0.26-1.11, $P=.10$, adjusted $P=.49$; Multimedia Appendix 9). Thrive intervention group participants were $45 \%$ and $58 \%$ less likely than controls to experience increased suicidal ideation following the entire 8-week follow-up period and at week 8 , respectively (Multimedia Appendix 9).

\section{Discussion}

\section{Principal Results}

This study evaluated the effectiveness of a fully automated, stand-alone, video-centric iCBT intervention (Thrive) for reducing depression symptom severity among adults residing in Montana. The design incorporated features of practical, pragmatic, and community-based effectiveness trials: liberal inclusion criteria (mild to severe depression symptoms), allowance for any past and concurrent treatments, and broad (symptom, functional, and resilience) outcomes. Over 8 weeks, depression symptom severity (primary outcome) in the Thrive intervention group was significantly reduced as compared to the control group. Additionally, the Thrive intervention group showed significant improvements in anxiety symptoms, work and social functioning, and resilience compared with the control group.

Although the Thrive intervention, like other iCBT interventions for depression in non-US populations [19], may be equally effective in rural and urban populations, the intervention has several characteristics that may make it appealing to rural US residents as an alternative or supplemental mental health intervention for depression symptoms. A previous qualitative study of Thrive among rural Montana adults led to an adaptation of 6 of the $>300$ videos to better reflect scenarios and settings common in rural communities [41], which may improve user engagement. Thrive can be performed in the privacy of one's home, which might offset mental health-related stigma, a frequent barrier to seeking mental health services experienced by rural US residents [51]. Thrive is accessible from any location with internet access and can be used on smartphones, tablets, and computers, making it an easily accessible, cost-effective alternative for rural residents who often have to drive long distances for in-person mental health care [41].

\section{Comparison With Prior Work}

To our knowledge, the clinical effect of the Thrive intervention on depression symptoms is one of the largest reported for an iCBT intervention delivered without clinician interaction. Omitting clinician support allows for broader generalizability to community populations by limiting costs and avoiding barriers associated with an underresourced mental health workforce. A recent meta-analysis of 16 self-guided iCBT studies by Karyotaki et al [21] and a systematic review by Lorenzo-Luaces et al [22] of the same 16 and an additional 5 self-guided iCBT studies reported that 7 studies evaluated iCBT interventions that, like Thrive, had no clinician contact [25-31]. The impact of Thrive on depression symptoms $(d=0.63)$ was greater than or similar to the 7 comparable studies (nonsignificant [25]; $d=0.17$ [26]; $d=0.20$ [28]; $d=0.28$ [27]; $d=0.30$ and 0.65 [for analysis of variance and mixed model analyses, respectively] [29]; $g=0.36$ [31]; and $d=0.50$ [30]). The greater impact of Thrive may be a result of the intervention group completing more lessons on average (8.7) than some other fully automated, stand-alone iCBT interventions [24-26].

Compared to self-guided iCBT interventions delivered with meaningful clinician support, described in Karyotaki et al [21] and Lorenzo-Luaces et al [22], the Thrive intervention yielded a similar or greater effect size for depression symptoms: Four studies that used clinician/research personnel contact to determine eligibility found effect sizes of $d=0.08$ [52], $d=0.38$ [53], $d=0.8$ [54], and $g=0.76$ for depression symptoms [55], although the latter study excluded "outliers" from the primary analysis and stated their inclusion resulted in "nonsignificant findings" and reduced the effect size by an undisclosed amount [55]. Two other studies that used iCBT in combination with contact with a therapist also reported similar or lower effect sizes ( $d=0.51$ [56] and 0.38 [31]) than those reported for Thrive. Six studies that included individualized email or SMS check-in reminders or telephone diagnostic interviews produced nonsignificant [57] or small-to-medium effect sizes for depression symptoms ( $d=0.34$ [30]; $d=0.36$ [58]; $d=0.55$ [59]; $d=0.57$ [60]; and $d=0.66$ [61]). Finally, the six studies providing up to weekly nonautomated email or telephone support reported no significant differences compared to the control for two studies [24,62] and small-to-large significant effects sizes for four studies: (1) $d=0.22$ and $d=0.34$ for support upon request and weekly support, respectively [26]; (2) $d=0.4$ (pre-post within-subjects effect size) [63]; (3) $d=0.39$ [64]; and (4) $d=1.14$ [61].

This study found similar or greater effect sizes for secondary outcomes (anxiety symptoms $[d=0.47]$, work and social functioning $[d=0.39]$, and resilience $[d=0.55])$ compared to the 21 iCBT trials described in the recent meta-analysis and systematic review [21,22]. In the seven studies reporting anxiety symptom outcomes, four identified nonsignificant differences with the control $[53,56,60,62]$ and three found small-to-nearly moderate effect sizes $(d=0.22$ [54], $d=0.25$ [26], and $d=0.43$ [30]). The three studies that assessed life/work functioning (using the same measure reported here [WSAS]) found a nonsignificant difference as compared to the control [62], a significant improvement for iCBT+treatment as usual but not in iCBT compared to the control (effect sizes not provided) 
[52], and an effect size of 0.36 [29]. Two other studies found no significant difference [57] and an effect size of 0.65 [54] when measuring life/work functioning with the Sheehan Disability Scale. To our knowledge, no other iCBT studies have evaluated resilience. Lastly, this study found a nonsignificant reduction $(58 \%)$ in the odds of having greater suicidal ideation in the Thrive intervention group than in the control group at 8 weeks. A small effect size $(d=0.20)$ was found in the single prior study that measured the impact of an iCBT intervention on suicidality [58].

The design of this study supports the generalizability of its findings in community settings. First, the minimal eligibility restrictions indicate the potential applicability of the Thrive intervention in general populations outside the controlled research setting. Of the seven studies described in Karyotaki et al [21] and Lorenzo-Luaces et al [22], only one that delivered a fully automated, stand-alone iCBT intervention like the Thrive intervention had similarly broad eligibility criteria [29]. Second, omitting clinician-administered diagnostic evaluations, as used in several iCBT studies [57,59-61], allows for broader generalizability to community settings. The use of person-administered diagnostics greatly increases costs, limits broad intervention dissemination due to an insufficient mental health workforce, and decreases the potential public health impact of fully automated, stand-alone iCBT interventions.

\section{Limitations}

This study had several limitations. Like most iCBT studies [21], assessment completion rates were low, with $68 \%$ and $65 \%$ of randomized subjects completing assessments at 4 and 8 weeks, respectively. These rates are slightly below the $73 \%$ (range $55 \%-95 \%)$ unweighted mean completion rate [24-30,52,55,58-64] for short-term (6-16 weeks) follow-up assessments of all studies in Karyotaki et al [21]. Additionally, the study offered monetary incentives for 4- and 8-week survey completion, which are associated with greater response in electronic questionnaires [65] and retention rates [66], which limits conclusions about uptake of Thrive in nonresearch settings. Monetary incentives have been used in all six RCTs of iCBT interventions for depression in US populations $[24,25,27,28,54,57]$ and three of the seven $(43 \%)$ RCTs of fully automated, stand-alone iCBT interventions reported in Karyotaki et al and Lorenzo-Luaces et al [21,22,25,27,28]. Although using self-assessments, a common practice in iCBT studies, is a potential weakness of the study, the use of validated, widely used instruments largely addressed this issue. The PHQ-9 (depression symptoms), Generalized Anxiety Disorders Scale-7 (anxiety symptoms), and WSAS-5 (functioning) correlate well with clinician-administered instruments $[37,67,68]$ and are sensitive to interventional effects $[45,69,70]$. Of note, self-assessments may underestimate the effect of iCBT relative to similar studies using clinician-administered assessments [71]. The study eligibility, which omitted the need for a depressive disorder diagnosis and included participants across the full range of depression symptom severity increases the study's generalizability. However, the make-up of the recruited study population, with high a proportion of female $(85 \%)$ and white (93\%) participants with at least some education after high school $(69 \%)$ limits its generalizability. Greater participation of women is a common limitation among studies of iCBT for depression. For example, Karyotaki et al [21] reported that $66 \%$ of all participants were female, and among the studies of fully automated, stand-alone iCBT interventions, female participation ranged from $65 \%$ to $81 \%$ [21]. The high proportion of female participants across iCBT studies could be due, in part, to the greater prevalence of depression in women [2] or as a yet-unidentified barrier for men. The relatively high percentage $(23.5 \%)$ of fraudulent participants identified is another limitation, which is becoming increasingly common in internet-based studies [72]. The number of fraudulent participants in this study is similar to that in several other studies using Web-based surveys: $28.7 \%$ [73], 20.5\% [74], and $18.7 \%$ [75]. Although the study was powered appropriately to detect between-group differences in a priori defined primary and secondary outcomes, even when allowing for corrections for multiple testing (design strength of this study), it was underpowered to detect meaningful difference in the post-hoc assessment of suicidal ideation, as only $41 \%$ of subjects experienced suicidality at baseline. In addition, the 8-week evaluation period cannot inform on the long-term impact of the Thrive intervention when delivered in community settings. Longitudinal studies with longer follow-up periods are warranted to determine whether iCBT programs impact the risk of depression relapse [10]. Finally, as a community-based trial, these findings cannot be generalized to clinical settings.

\section{Conclusions}

Thrive, a fully automated, stand-alone iCBT intervention, demonstrated greater short-term improvements in depression and anxiety symptoms, work and social functioning, and resilience compared to control participants in a broad community population with mild to severe depression symptoms. A trend toward greater reduction in suicidal ideation was also observed. The magnitude of clinical benefit seen with the Thrive intervention appears to be greater than most other fully automated, stand-alone interventions and similar to those requiring clinician support. Thus, Thrive represents a potentially effective and cost-effective solution for treating depression symptoms in rural regions that often have few or no mental health providers. Evaluations of the impact of the Thrive intervention over longer periods of time and other population settings are warranted.

\section{Acknowledgments}

Research reported in this publication was supported by the National Institute of General Medical Sciences of the National Institutes of Health (P20GM103474, U54GM115371, and 5P20GM104417). The content is solely the responsibility of the authors and does not necessarily represent the official views of the National Institutes of Health.

At the time of the trial, JL and MB were affiliated with the Center for Mental Health Research and Recovery at Montana State University, Bozeman, Montana. JL is currently affiliated with the Department of Psychiatry at the University of Arizona, the 
Biomedical Research and Education Foundation of Southern Arizona, and the Southern Arizona VA Health Care System, Tucson, Arizona. MB is currently affiliated with the Department of Psychiatry at the University of Arizona and the Southern Arizona VA Health Care System, Tucson, Arizona.

\section{Conflicts of Interest}

JHG previously held a financial interest in Waypoint Health Innovations, which developed the Thrive intervention evaluated in this work. He no longer has a direct financial interest in Waypoint Health Innovations but does retain a small interest in Waypoint Health Innovations through Healthcare Technology Systems where he is CEO and a shareholder. Waypoint Health Innovations also pays him a royalty based on revenue from Thrive use. He is also a consultant to Waypoint on projects outside of the grant supporting this study. The terms of JHG's financial relationship with Waypoint Health Innovations have been reviewed by Montana State University, and his involvement with this research project has been approved in accordance with its conflict of interest policies.

\section{Multimedia Appendix 1}

Informed consent.

[DOCX File, $21 \mathrm{~KB}-$ Multimedia Appendix 1]

\section{Multimedia Appendix 2}

Description of study population with definition and removal of fraudulent participants.

[DOCX File, $30 \mathrm{~KB}$-Multimedia Appendix 2]

\section{Multimedia Appendix 3}

CONSORT diagram.

[DOCX File , $64 \mathrm{~KB}$-Multimedia Appendix 3]

\section{Multimedia Appendix 4}

Study safety protocol.

[DOCX File, 42 KB-Multimedia Appendix 4]

\section{Multimedia Appendix 5}

Covariates.

[DOCX File, 23 KB-Multimedia Appendix 5]

\section{Multimedia Appendix 6}

Power calculations.

[DOCX File, 34 KB-Multimedia Appendix 6]

\section{Multimedia Appendix 7}

Baseline PHQ-9 scores.

[DOCX File, 14 KB-Multimedia Appendix 7]

\section{Multimedia Appendix 8}

Adjusted least mean squares for secondary outcomes.

[PNG File, 539 KB-Multimedia Appendix 8]

\section{Multimedia Appendix 9}

Effect of the Thrive intervention on suicidal ideation.

[DOCX File, 36 KB-Multimedia Appendix 9]

\section{Multimedia Appendix 10}

Participant flow chart.

[DOCX File, 78 KB-Multimedia Appendix 10] 


\section{Multimedia Appendix 11}

CONSORT - EHEALTH checklist (V 1.6.1).

[PDF File (Adobe PDF File), 352 KB-Multimedia Appendix 11]

\section{References}

1. World Health Organization. 2017. Depression and other common mental disorders: global health estimates URL: https:/ lapps.who.int/iris/bitstream/handle/10665/254610/WHO-MSD-MER-2017.2-eng.pdf [accessed 2019-11-08]

2. Hasin DS, Sarvet AL, Meyers JL, Saha TD, Ruan WJ, Stohl M, et al. Epidemiology of Adult DSM-5 Major Depressive Disorder and Its Specifiers in the United States. JAMA Psychiatry 2018 Apr 01;75(4):336-346 [FREE Full text] [doi: 10.1001/jamapsychiatry.2017.4602] [Medline: 29450462]

3. Gilman SE, Sucha E, Kingsbury M, Horton NJ, Murphy JM, Colman I. Depression and mortality in a longitudinal study: 1952-2011. CMAJ 2017 Oct 23;189(42):E1304-E1310 [FREE Full text] [doi: 10.1503/cmaj.170125] [Medline: 29061855]

4. Cuijpers P, Vogelzangs N, Twisk J, Kleiboer A, Li J, Penninx BW. Comprehensive meta-analysis of excess mortality in depression in the general community versus patients with specific illnesses. Am J Psychiatry 2014 Apr;171(4):453-462. [doi: 10.1176/appi.ajp.2013.13030325] [Medline: 24434956]

5. Berghöfer A, Roll S, Bauer M, Willich SN, Pfennig A. Screening for depression and high utilization of health care resources among patients in primary care. Community Ment Health J 2014 Oct;50(7):753-758. [doi: 10.1007/s10597-014-9700-4] [Medline: 24449430]

6. Chesney E, Goodwin GM, Fazel S. Risks of all-cause and suicide mortality in mental disorders: a meta-review. World Psychiatry 2014 Jun;13(2):153-160 [FREE Full text] [doi: 10.1002/wps.20128] [Medline: 24890068]

7. Biesheuvel-Leliefeld KEM, Kok GD, Bockting CLH, Cuijpers P, Hollon SD, van Marwijk HWJ, et al. Effectiveness of psychological interventions in preventing recurrence of depressive disorder: meta-analysis and meta-regression. J Affect Disord 2015 Mar 15;174:400-410. [doi: 10.1016/j.jad.2014.12.016] [Medline: 25553400]

8. Vittengl JR, Clark LA, Dunn TW, Jarrett RB. Reducing relapse and recurrence in unipolar depression: a comparative meta-analysis of cognitive-behavioral therapy's effects. J Consult Clin Psychol 2007 Jun;75(3):475-488 [FREE Full text] [doi: 10.1037/0022-006X.75.3.475] [Medline: 17563164]

9. Chand S, Chaitanya R, Chakkamparambil B, Prasad A, Vora A. CBT for depresssion: what the evidence says. Curr Psychiat 2018;17(9):14-23.

10. Webb CA, Rosso IM, Rauch SL. Internet-Based Cognitive-Behavioral Therapy for Depression: Current Progress and Future Directions. Harv Rev Psychiatry 2017;25(3):114-122 [FREE Full text] [doi: 10.1097/HRP.0000000000000139] [Medline: $\underline{28475503}$ ]

11. United States Census Bureau. 2016. New census data show differences between urban and rural populations URL: $\underline{\text { https:/ }}$ /www.census.gov/newsroom/press-releases/2016/cb16-210.html [accessed 2019-06-01]

12. Kaiser Family Foundation. 2018 Sep 5. Mental Health Care Health Professional Shortage Areas (HPSAs) URL: https:/ /tinyurl.com/ygtbh8w4 [accessed 2019-09-18]

13. US Department of Agriculture. State fact sheets: Montana URL: https://data.ers.usda.gov/reports. aspx?StateFIPS=30\&StateName=Montana $\&$ ID=17854 [accessed 2019-09-18]

14. Centers for Disease Control and Prevention. 2017. BRFSS prevalence \& trends data URL: https://www.cdc.gov/brfss/ brfssprevalence [accessed 2019-09-18]

15. Centers for Disease Control and Prevention. 2018. Suicide Mortality by State URL: https://www.cdc.gov/nchs/pressroom/ sosmap/suicide-mortality/suicide.htm [accessed 2019-09-18]

16. Andersson G, Cuijpers P. Internet-based and other computerized psychological treatments for adult depression: a meta-analysis. Cogn Behav Ther 2009 Dec;38(4):196-205. [doi: 10.1080/16506070903318960] [Medline: 20183695]

17. Andersson G, Cuijpers P, Carlbring P, Riper H, Hedman E. Guided Internet-based vs. face-to-face cognitive behavior therapy for psychiatric and somatic disorders: a systematic review and meta-analysis. World Psychiatry 2014 Oct;13(3):288-295 [FREE Full text] [doi: 10.1002/wps.20151] [Medline: 25273302]

18. Andrews G, Basu A, Cuijpers P, Craske MG, McEvoy P, English CL, et al. Computer therapy for the anxiety and depression disorders is effective, acceptable and practical health care: An updated meta-analysis. J Anxiety Disord 2018 Apr;55:70-78 [FREE Full text] [doi: 10.1016/j.janxdis.2018.01.001] [Medline: 29422409]

19. Vallury KD, Jones M, Oosterbroek C. Computerized Cognitive Behavior Therapy for Anxiety and Depression in Rural Areas: A Systematic Review. J Med Internet Res 2015;17(6):e139 [FREE Full text] [doi: 10.2196/jmir.4145] [Medline: 26048193]

20. Statista. 2017 Mar 11. U.S. home broadband penetration by state URL: https://www.statista.com/chart/10600/ us-home-broadband-penetration-by-state/ [accessed 2019-09-18]

21. Karyotaki E, Riper H, Twisk J, Hoogendoorn A, Kleiboer A, Mira A, et al. Efficacy of Self-guided Internet-Based Cognitive Behavioral Therapy in the Treatment of Depressive Symptoms: A Meta-analysis of Individual Participant Data. JAMA Psychiatry 2017 Apr 01;74(4):351-359. [doi: 10.1001/jamapsychiatry.2017.0044] [Medline: 28241179] 
22. Lorenzo-Luaces L, Johns E, Keefe JR. The Generalizability of Randomized Controlled Trials of Self-Guided Internet-Based Cognitive Behavioral Therapy for Depressive Symptoms: Systematic Review and Meta-Regression Analysis. J Med Internet Res 2018 Nov 09;20(11):e10113 [FREE Full text] [doi: 10.2196/10113] [Medline: 30413400]

23. Richards D, Richardson T. Computer-based psychological treatments for depression: a systematic review and meta-analysis. Clin Psychol Rev 2012 Jun;32(4):329-342. [doi: 10.1016/j.cpr.2012.02.004] [Medline: 22466510]

24. Gilbody S, Littlewood E, Hewitt C, Brierley G, Tharmanathan P, Araya R, et al. Computerised cognitive behaviour therapy (cCBT) as treatment for depression in primary care (REEACT trial): large scale pragmatic randomised controlled trial. BMJ 2015;351:h5627 [FREE Full text] [doi: 10.1136/bmj.h5627] [Medline: 26559241]

25. Clarke G, Reid E, Eubanks D, O'Connor E, DeBar LL, Kelleher C, et al. Overcoming depression on the Internet (ODIN): a randomized controlled trial of an Internet depression skills intervention program. J Med Internet Res 2002 Dec;4(3):E14 [FREE Full text] [doi: 10.2196/jmir.4.3.e14] [Medline: 12554545]

26. Kleiboer A, Donker T, Seekles W, van Straten A, Riper H, Cuijpers P. A randomized controlled trial on the role of support in Internet-based problem solving therapy for depression and anxiety. Behav Res Ther 2015 Sep;72:63-71. [doi: 10.1016/j.brat.2015.06.013] [Medline: 26188373]

27. Clarke G, Eubanks D, Reid E, Kelleher C, O'Connor E, DeBar LL, et al. Overcoming Depression on the Internet (ODIN) (2): a randomized trial of a self-help depression skills program with reminders. J Med Internet Res 2005;7(2):e16 [FREE Full text] [doi: 10.2196/jmir.7.2.e16] [Medline: 15998607]

28. Clarke G, Kelleher C, Hornbrook M, Debar L, Dickerson J, Gullion C. Randomized effectiveness trial of an Internet, pure self-help, cognitive behavioral intervention for depressive symptoms in young adults. Cogn Behav Ther 2009;38(4):222-234 [FREE Full text] [doi: 10.1080/16506070802675353] [Medline: 19440896]

29. Meyer B, Berger T, Caspar F, Beevers CG, Andersson G, Weiss M. Effectiveness of a novel integrative online treatment for depression (Deprexis): randomized controlled trial. J Med Internet Res 2009;11(2):e15 [FREE Full text] [doi: 10.2196/jmir.1151] [Medline: 19632969]

30. Mira A, Bretón-López J, García-Palacios A, Quero S, Baños RM, Botella C. An Internet-based program for depressive symptoms using human and automated support: a randomized controlled trial. Neuropsychiatr Dis Treat 2017;13:987-1006 [FREE Full text] [doi: 10.2147/NDT.S130994] [Medline: 28408833]

31. Montero-Marín J, Araya R, Pérez-Yus MC, Mayoral F, Gili M, Botella C, et al. An Internet-Based Intervention for Depression in Primary Care in Spain: A Randomized Controlled Trial. J Med Internet Res 2016;18(8):e231 [FREE Full text] [doi: 10.2196/jmir.5695] [Medline: 27565118]

32. Beck A, Rush A, Shaw B, Emery G. Cognitive Therapy Of Depression (the Guilford Clinical Psychology And Psychopathology Series). New York, NY: The Guilford Press; 1979.

33. Lewinsohn P, Munoz R, Youngren M, Zeiss A. Control Your Depression. Englewood Cliffs, NJ: Prentice-Hall Press; 1986.

34. Cuijpers P, van Straten A, Warmerdam L. Behavioral activation treatments of depression: a meta-analysis. Clin Psychol Rev 2007 Apr;27(3):318-326. [doi: 10.1016/j.cpr.2006.11.001] [Medline: 17184887]

35. Alberti R, Emmons M. Your Perfect Right: Assertiveness And Equality In Your Life And Relationships, 10th Edition. Oakland, CA: New Harbinger Publications; 2017.

36. Whiteside U, Richards J, Steinfeld B, Simon G, Caka S, Tachibana C, et al. Online cognitive behavioral therapy for depressed primary care patients: a pilot feasibility project. Perm J 2014 May;18(2):21-27 [FREE Full text] [doi: 10.7812/TPP/13-155] [Medline: 24867546]

37. Kroenke K, Spitzer RL, Williams JB. The PHQ-9: validity of a brief depression severity measure. J Gen Intern Med 2001 Sep;16(9):606-613 [FREE Full text] [doi: 10.1046/j.1525-1497.2001.016009606.x] [Medline: 11556941]

38. United States Census Bureau. Quick facts: Montana URL: https://www.census.gov/quickfacts/MT [accessed 2019-09-18]

39. Montana State University. 2018. Thrive for Montana study URL: https://thriveformontana.com/ [accessed 2019-11-08]

40. Waypoint Health Innovations. Waypoint Health Innovations. 2018. Thrive URL: https://waypointhealth.com/thrive/ [accessed 2019-11-08]

41. Schure MB, Howard M, Bailey SJ, Bryan B, Greist J. Exploring Perceptions of a Computerized Cognitive Behavior Therapy Program in a U.S. Rural Western State. Rural Ment Health 2018 Jul;42(3-4):174-183. [doi: 10.1037/rmh0000102] [Medline: $\underline{30740195]}$

42. National Institute of Mental Health. 2019. Depression URL: https://www.nimh.nih.gov/health/topics/depression [accessed 2019-09-18]

43. National Alliance on Mental Illness - Montana. 2019. County Mental Illness Resource Guides URL: http://www.namimt.org/ county-resource-guides.html [accessed 2019-09-18]

44. Löwe B, Decker O, Müller S, Brähler E, Schellberg D, Herzog W, et al. Validation and standardization of the Generalized Anxiety Disorder Screener (GAD-7) in the general population. Med Care 2008 Mar;46(3):266-274. [doi: 10.1097/MLR.0b013e318160d093] [Medline: 18388841]

45. Mundt JC, Marks IM, Shear MK, Greist JM. The Work and Social Adjustment Scale: a simple measure of impairment in functioning. Br J Psychiatry 2002 May;180:461-464. [doi: 10.1192/bjp.180.5.461] [Medline: 11983645] 
46. Campbell-Sills L, Stein MB. Psychometric analysis and refinement of the Connor-davidson Resilience Scale (CD-RISC): Validation of a 10-item measure of resilience. J Trauma Stress 2007 Dec;20(6):1019-1028. [doi: 10.1002/jts.20271] [Medline: $\underline{18157881]}$

47. Wolfinger R, Chang M. Comparing the SAS GLM and MIXED procedures for repeated measures. Presented at: Proceedings of the Twentieth Annual SAS Users Group Conference; 1998; Cary, NC.

48. Little RJ. Modeling the Drop-Out Mechanism in Repeated-Measures Studies. Journal of the American Statistical Association 1995 Sep;90(431):1112-1121. [doi: 10.2307/2291350]

49. Rubin D. Inference and missing data. Biometrika 1976 Dec;63(3):581-592. [doi: 10.1093/biomet/63.3.581]

50. Documentation: United States Department of Agriculture. 2019. 2010 Rural-Urban Communiting Area (RUCA) Codes URL: https://www.ers.usda.gov/data-products/rural-urban-commuting-area-codes/documentation/ [accessed 2019-09-18]

51. Larson JE, Corrigan PW. Psychotherapy for self-stigma among rural clients. J Clin Psychol 2010 May;66(5):524-536. [doi: 10.1002/jclp.20679] [Medline: 20222119]

52. de Graaf LE, Gerhards SAH, Arntz A, Riper H, Metsemakers JFM, Evers SMAA, et al. Clinical effectiveness of online computerised cognitive-behavioural therapy without support for depression in primary care: randomised trial. Br J Psychiatry 2009 Jul;195(1):73-80. [doi: 10.1192/bjp.bp.108.054429] [Medline: 19567900 ]

53. Terides MD, Dear BF, Fogliati VJ, Gandy M, Karin E, Jones MP, et al. Increased skills usage statistically mediates symptom reduction in self-guided internet-delivered cognitive-behavioural therapy for depression and anxiety: a randomised controlled trial. Cogn Behav Ther 2018 Jan;47(1):43-61. [doi: 10.1080/16506073.2017.1347195] [Medline: 28724338]

54. Beevers CG, Pearson R, Hoffman JS, Foulser AA, Shumake J, Meyer B. Effectiveness of an internet intervention (Deprexis) for depression in a united states adult sample: A parallel-group pragmatic randomized controlled trial. J Consult Clin Psychol 2017 Apr;85(4):367-380. [doi: 10.1037/ccp0000171] [Medline: 28230390]

55. Farrer L, Christensen H, Griffiths KM, Mackinnon A. Internet-based CBT for depression with and without telephone tracking in a national helpline: randomised controlled trial. PLoS One 2011;6(11):e28099 [FREE Full text] [doi: 10.1371/journal.pone.0028099] [Medline: 22140514]

56. Berger T, Krieger T, Sude K, Meyer B, Maercker A. Evaluating an e-mental health program ("deprexis") as adjunctive treatment tool in psychotherapy for depression: Results of a pragmatic randomized controlled trial. J Affect Disord 2018 Feb;227:455-462. [doi: 10.1016/j.jad.2017.11.021] [Medline: 29154168]

57. Arean PA, Hallgren KA, Jordan JT, Gazzaley A, Atkins DC, Heagerty PJ, et al. The Use and Effectiveness of Mobile Apps for Depression: Results From a Fully Remote Clinical Trial. J Med Internet Res 2016 Dec 20;18(12):e330 [FREE Full text] [doi: 10.2196/jmir.6482] [Medline: 27998876]

58. Moritz S, Schilling L, Hauschildt M, Schröder J, Treszl A. A randomized controlled trial of internet-based therapy in depression. Behav Res Ther 2012 Aug;50(7-8):513-521. [doi: 10.1016/j.brat.2012.04.006] [Medline: 22677231]

59. Spek V, Nyklícek I, Smits N, Cuijpers P, Riper H, Keyzer J, et al. Internet-based cognitive behavioural therapy for subthreshold depression in people over 50 years old: a randomized controlled clinical trial. Psychol Med 2007 Dec;37(12):1797-1806. [doi: 10.1017/S0033291707000542] [Medline: 17466110]

60. Meyer B, Bierbrodt J, Schröder J, Berger T, Beevers CG, Weiss M, et al. Effects of an Internet intervention (Deprexis) on severe depression symptoms: Randomized controlled trial. Internet Interventions 2015 Mar;2(1):48-59. [doi: 10.1016/j.invent.2014.12.003]

61. Berger T, Hämmerli K, Gubser N, Andersson G, Caspar F. Internet-based treatment of depression: a randomized controlled trial comparing guided with unguided self-help. Cogn Behav Ther 2011;40(4):251-266. [doi: 10.1080/16506073.2011.616531] [Medline: 22060248]

62. Phillips R, Schneider J, Molosankwe I, Leese M, Foroushani PS, Grime P, et al. Randomized controlled trial of computerized cognitive behavioural therapy for depressive symptoms: effectiveness and costs of a workplace intervention. Psychol Med 2014 Mar;44(4):741-752 [FREE Full text] [doi: 10.1017/S0033291713001323] [Medline: 23795621]

63. Christensen H, Griffiths KM, Jorm AF. Delivering interventions for depression by using the internet: randomised controlled trial. BMJ 2004 Jan 31;328(7434):265 [FREE Full text] [doi: 10.1136/bmj.37945.566632.EE] [Medline: 14742346]

64. Klein JP, Berger T, Schröder J, Späth C, Meyer B, Caspar F, et al. Effects of a Psychological Internet Intervention in the Treatment of Mild to Moderate Depressive Symptoms: Results of the EVIDENT Study, a Randomized Controlled Trial. Psychother Psychosom 2016;85(4):218-228. [doi: 10.1159/000445355] [Medline: 27230863]

65. Brueton VC, Tierney JF, Stenning S, Meredith S, Harding S, Nazareth I, et al. Strategies to improve retention in randomised trials: a Cochrane systematic review and meta-analysis. BMJ Open 2014 Feb 04;4(2):e003821 [FREE Full text] [doi: 10.1136/bmjopen-2013-003821] [Medline: 24496696]

66. Liu Y, Pencheon E, Hunter RM, Moncrieff J, Freemantle N. Recruitment and retention strategies in mental health trials A systematic review. PLoS One 2018;13(8):e0203127 [FREE Full text] [doi: 10.1371/journal.pone.0203127] [Medline: 30157250]

67. Löwe B, Kroenke K, Herzog W, Gräfe K. Measuring depression outcome with a brief self-report instrument: sensitivity to change of the Patient Health Questionnaire (PHQ-9). J Affect Disord 2004 Jul;81(1):61-66. [doi:

10.1016/S0165-0327(03)00198-8] [Medline: 15183601] 
68. Spitzer RL, Kroenke K, Williams JBW, Löwe B. A brief measure for assessing generalized anxiety disorder: the GAD-7. Arch Intern Med 2006 May 22;166(10):1092-1097. [doi: 10.1001/archinte.166.10.1092] [Medline: 16717171]

69. Zimmerman M, Walsh E, Friedman M, Boerescu DA, Attiullah N. Are self-report scales as effective as clinician rating scales in measuring treatment response in routine clinical practice? J Affect Disord 2018 Jan 01;225:449-452. [doi: 10.1016/j.jad.2017.08.024] [Medline: 28858659]

70. Kertz S, Bigda-Peyton J, Bjorgvinsson T. Validity of the Generalized Anxiety Disorder-7 scale in an acute psychiatric sample. Clin Psychol Psychother 2013;20(5):456-464. [doi: 10.1002/cpp.1802] [Medline: 22593009]

71. Cuijpers P, Li J, Hofmann SG, Andersson G. Self-reported versus clinician-rated symptoms of depression as outcome measures in psychotherapy research on depression: a meta-analysis. Clin Psychol Rev 2010 Aug;30(6):768-778. [doi: 10.1016/j.cpr.2010.06.001] [Medline: 20619943]

72. Teitcher JEF, Bockting WO, Bauermeister JA, Hoefer CJ, Miner MH, Klitzman RL. Detecting, preventing, and responding to "fraudsters" in internet research: ethics and tradeoffs. J Law Med Ethics 2015;43(1):116-133 [FREE Full text] [doi: 10.1111/jlme.12200] [Medline: 25846043]

73. Ballard AM, Cardwell T, Young AM. Fraud Detection Protocol for Web-Based Research Among Men Who Have Sex With Men: Development and Descriptive Evaluation. JMIR Public Health Surveill 2019 Feb 04;5(1):e12344 [FREE Full text] [doi: 10.2196/12344] [Medline: 30714944]

74. Bull S, Pratte K, Whitesell N, Rietmeijer C, McFarlane M. Effects of an Internet-based intervention for HIV prevention: the Youthnet trials. AIDS Behav 2009 Jun;13(3):474-487 [FREE Full text] [doi: 10.1007/s10461-008-9487-9] [Medline: 19037719]

75. Bauermeister J, Pingel E, Zimmerman M, Couper M, Carballo-Diéguez A, Strecher VJ. Data Quality in web-based HIV/AIDS research: Handling Invalid and Suspicious Data. Field methods 2012 Aug 01;24(3):272-291 [FREE Full text] [doi: 10.1177/1525822X12443097] [Medline: 23180978]

\author{
Abbreviations \\ CDRISC-10: Connor Davidson Resilience Scale-10 \\ DSM: Diagnostic and Statistical Manual of Mental Disorders \\ GAD-7: Generalized Anxiety Disorder 7-item scale \\ iCBT: internet-based cognitive behavior therapy \\ LSM: least squares means \\ OR: odds ratio \\ PHQ-9: Patient Health Questionnaire-9 \\ RCT: randomized controlled trial \\ WSAS-5: Work and Social Adjustment Scale-5
}

Edited by G Eysenbach; submitted 18.05.19; peer-reviewed by S Rauch, V Cosgrove, D Gratzer; comments to author $21.08 .19 ;$ revised
version received 23.09.19; accepted 22.10.19; published 18.11 .19
Please cite as:
Schure MB, Lindow JC, Greist JH, Nakonezny PA, Bailey SJ, Bryan WL, Byerly MJ
Use of a Fully Automated Internet-Based Cognitive Behavior Therapy Intervention in a Community Population of Adults With
Depression Symptoms: Randomized Controlled Trial
J Med Internet Res 2019;21(11):e14754
URL: $\underline{\text { http://www.jmir.org/2019/11/e14754/ }}$
doi: $\underline{10.2196 / 14754}$
PMID: $\underline{31738173}$

CMark B Schure, Janet C Lindow, John H Greist, Paul A Nakonezny, Sandra J Bailey, William L Bryan, Matthew J Byerly. Originally published in the Journal of Medical Internet Research (http://www.jmir.org), 18.11.2019. This is an open-access article distributed under the terms of the Creative Commons Attribution License (https://creativecommons.org/licenses/by/4.0/), which permits unrestricted use, distribution, and reproduction in any medium, provided the original work, first published in the Journal of Medical Internet Research, is properly cited. The complete bibliographic information, a link to the original publication on http://www.jmir.org/, as well as this copyright and license information must be included. 